\title{
Human-Machine Interface Evaluation of CNC Machine Control Panel through Multidimensional Experimental Data Synchronous Testing Analysis Method
}

\author{
Jinhua Dou ${ }^{\mathrm{a}, \mathrm{b}, *}$, Lei Zhang ${ }^{\mathrm{b}}$, Qichao Zhao ${ }^{\mathrm{c}}$, Qin Pei ${ }^{\mathrm{b}}$, Jingyan Qin ${ }^{\mathrm{a}, *}$ \\ ${ }^{a}$ School of Computer and Communication Engineering, School of Mechanical Engineering, University of Science and Technology Beijing, China \\ ${ }^{b}$ School of Art \& Design, Tianjin University of Technology, China \\ ${ }^{c}$ KingFar International Inc, Beijing, China
}

\begin{abstract}
Human-Machine Interface (HMI) of Computer Numerical Control (CNC) machine control panel affects the work efficiency and experience of users. For many operators, the CNC machine control panel causes a heavy burden for them because it is not easy to use. The designer creates the shape, color and layout of CNC machine control panel to meet the user's needs. There is some design schemes produced for managers or designers to make choices. Effective design evaluation can help the designer find usability issues of humanmachine interface and iterative optimization schemes. In this study, a practical exploration relating to HMI of CNC machine control panel was carried out using the multidimensional experimental data synchronous testing analysis method. Four HMI design schemes of CNC machine control panel were evaluated. The physiological measurement instruments, eye tracker and behavior analyzer were adopted to obtain the user's physiological data, psychological data and behavioral data. Scientific statistical results were formed and output by visualization. The designer can choose the best schemes and find the design elements to be improved by this mutually validating method. It's helpful for the designer to improve the ergonomics of human-machine interface design of CNC machine control panel.
\end{abstract}

Keywords: synchronous testing analysis; multidimensional experimental data; Human-Machine Interface (HMI); design evaluation; Computer Numerical Control (CNC); machine control panel

(Submitted on September 20, 2017; Revised on November 10, 2017; Accepted on November 30, 2017)

(C) 2017 Totem Publisher, Inc. All rights reserved.

\section{Introduction}

Computer Numerical Control (CNC) is widely used in various industries fields and it is important for modern standards of industrial manufacturing [29]. The CNC machine control panel is an important component of CNC machine tools. The Human-Machine Interface (HMI) is the user interface that people can access and communicate with a machine or device [14]. The HMI of CNC machine control panel affects the operation efficiency and experience of users. The operator can control the CNC machine system using the control panel to achieve mechanical parts processing, cutting and other operations. The control panel mainly includes total control area, pattern selection area, spindle control area, program edit area, and tool changer area.

For many operators, the CNC machine control panel poses a heavy burden for them because it is not easy to use. This is because poor design of HMI scheme doesn't conform to the user's mental model [13]. Designers create the shape and layout of CNC machine control panel to meet the user's needs. In the design process, some design schemes are often produced for managers or designers to make choices. It is very important to evaluate the different design schemes to help designers or managers select the best scheme. They can also find the usability issues of CNC machine control panel and optimize the 
design schemes through design evaluation. Therefore, the design evaluation of human-machine interface is necessary to assist in the design decisions and optimization of product schemes.

Experimental testing is an important design evaluation method for the product conceptual design [7]. In the process of experimental testing, the reliable conclusions are not easy to obtain through single-dimensional experimental testing data. Once the data analysis in one experiment doesn't reach the desired conclusions, the researchers need to examine the issues during the whole experiment process. They need consider whether the experimental design is unreasonable, if the experimental control is not strict, or if there are other interference factors. So, they need to carry out the repeated experiments to verify [7,18,30,33]. Therefore, some more accurate and effective methods are needed to solve the above problems. A comprehensive, multidimensional and synchronously experiment testing method is carried out to obtain different types of continuously monitored data. This method is superior to the traditional method since it can reveal the truer and more comprehensive evidence [9].

We proposed multidimensional experimental data synchronous testing analysis method to evaluate the design schemes. Physiology, psychology and behaviour are the three measurement dimensions in this study. We synchronously acquired data from these three dimensions through some measuring devices. The physiological measurement instruments, eye tracker and behavior analyzer were adopted to obtain usability issues of HMI concept design. Four design schemes of CNC machine control panel were produced. There were different function zones, key shapes and layouts. Designers can select the best schemes and find usability problems of these schemes using multidimensional experimental data synchronous testing analysis method.

The rest of the paper is organized as follows: Section 2 introduces the related work and research method of design evaluation. In section 3, the method in this study is presented and related work is introduced. In section 4, a detailed introduction is presented to explain the experimental objectives, equipment and tasks. In section 5, the testing data is acquired, identified, analyzed and the results are discussed. In section 6, some conclusions are given.

\section{Related works}

The experimental measurements of user's physiological, psychological and behavioral characteristics are the important research methods of HMI design evaluation. However, the experimental evaluations on the HMI of CNC machine control panel are very few. Therefore, we reviewed the experimental evaluation methods in some related fields.

Niu et al. [22] applied event-related potential (ERP) technique and behavioral data to study the cognition and evaluation of digital interface with different layout forms. Sinharay et al. [31] analyzed different onscreen keyboard layouts using Electroencephalogram (EEG) signals. Masaki et al. [17] evaluated the software-use experiences quantitatively using EEG.

Açık et al. [1] evaluated two different arrangements for the layout of the same surgical interfaces record using the eye movements. Darwish and Bataineh [6] presented the first empirical study of user interaction with web browsers' security indicators in a controlled real life security risk. Zhou et al. [35] compared the user interface arrangement of two games using eye tracking technology, they verified that the user interface arrangement was an important factor for game design. Ramakrisnan et al. [26] found some interface design issues of leaning management system by analyzing student's gaze plot and heat map of eye tracking pattern.

Akyeampong et al. [2] evaluated the newly human-machine interface design concepts based on augmented interaction techniques to improve the ergonomics of hydraulic excavators. Park and Moon [24] proposed an approach of design evaluation based on tangible augmented reality.

The above-mentioned research methods are scientific; however, they also have some disadvantages. The user's physiological data is vulnerable to external factors [7]. There are some problems in the study of eye movement. For example, the eye movement behavior is inconsistent with the mental states of the user, and the eye movement data is not objective enough [33]. Virtual reality evaluation also has many problems that need to be solved, including caused uncomfortable feeling and the lack of uniform standards [30].

Dirican et al. [7] aimed at studying human cognitive states. They reviewed psychophysiological measures applied in Human Computer Interaction (HCI). Giraudet et al. [10] proposed the approach of neuroergonomics to evaluate notification designs using behavioral, subjective and physiological results, so that people could more in-depth understand the complex impact of changes in interface design. Lin and Zhang [16] presented a study on a more objective evaluation technique based 
on the combination of operators' eye movement and hand movement behaviors. Andreoni et al. [4] evaluated portable ultrasound systems user interfaces using motion analysis and eye tracking technologies. They described the ergonomics and usability preliminary tests regarding the human upper limb kinematics and cognitive evaluation of two diagnostic portable ultrasound scanners. C. Guo [8] studied the synchronous record and analysis of eye movement and electroencephalograph. She proposed and achieved some key technologies. F. Guo et al. [11] studied the user's emotional experience and behavior intention when they browsed e-commerce sites by the physiological measurement, eye tracking and questionnaire survey methods.

The synchronous testing analysis based on the multidimensional data of physiological, psychological and behavioral can quickly find the root of the problem. Researchers can quickly find the interference factors, which affect the accuracy of experimental results. For the experiment to be designed reasonably, most of the indicators can be fully explained. A small number of conflicting indicators will not seriously affect the accuracy of the analysis results. When multidimensional experimental data presents the unexplained contradictory situations, the researchers need to rethink the rationality of the experimental design if it's not caused by single interference factor. So, the synchronous testing and analysis method of multidimensional data will make the evaluation result more objectively. In this study, a practical exploration relating to HMI of CNC machine control panel was carried out using multidimensional experiment data synchronous testing analysis method.

\section{Method}

\subsection{Construction of virtual model for design concept}

According to the product analysis and user research, the HMI of CNC machine control panel was designed and the 3D digital model was constructed using three-dimensional modeling software. The shape, location and layout of button were shown with the virtual scene of three-dimensional products simulation. The experimental environment was constructed and a multidimensional data synchronous testing platform was built.

\subsection{Multidimensional data synchronously acquisition in the process of user interaction with CNC machine control panel}

Multidimensional data synchronous testing method can simultaneously record the multi-parameter data at the same time point and same time period. The start / stop points of event and each data are synchronously triggered, which can be accurate to the milliseconds in time. Physiological data e.g., electroencephalogram (EEG), electrocardiogram (ECG), skin conductivity (SC), eye movement, actions and facial expressions are synchronously acquired. The combinations of human internal physiological feedback and explicit behavior are helpful to get more accurate conclusions. At present, the main measurement modules are the physiological measurement module, eye tracking measurement module, behavior observation module and physical environment measurement module. In this study, some measurement modules were selected according to the different experimental objectives, so that they could match the sample attribution definition and provide the basis for further research.

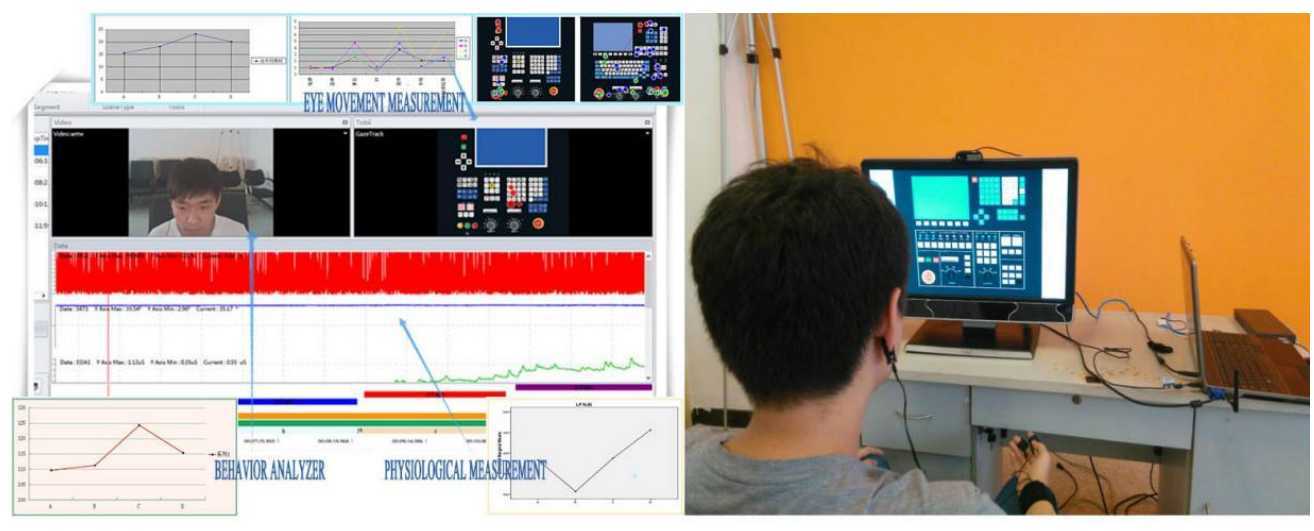

Figure 1. Multidimensional data synchronous testing analysis methods

The process of multidimensional data synchronously acquisition is shown as follows:

- $\quad$ Physiological data acquisition. The user's physiological index, e.g., Electrodermal Activity (EDA), Heart Rate Variability (HRV) was obtained using appropriate physiological sensors [3,23]. 
- Eye movement data acquisition. The user's psychological characteristics and behavior were investigated in terms of the method of eye tracking.

- Behavior data testing acquisition. The detailed behavior reactions and task executive process were collected using eye movement experiment and behavior observation measurement.

Tobi IT X300 desktop eye tracker, pulse physiological sensors, skin physiological sensors and behavior analysis camera were used to real time synchronously measure the user's response. The physiological response, eye movement and body actions of users were recorded. The physiological and psychological data were obtained synchronously at the same time period as shown in Figure 1.

\subsection{Design evaluation based on multidimensional data synchronous analysis}

The multidimensional data obtained from testing experiments have been processed, analyzed and mined. The specific data fragments or meaningful data were selected as the analyzing object. The physiological and psychological characteristics of users were analyzed with the multidimensional data. The scientific statistical results would be formed and output by visualization. Some contradictory results that were not confirmed with each other could be reasonably explained. The results could be verified with each other by multidimensional data synchronous analysis. So, we could evaluate the HMI of CNC machine control panel design more objectively.

\section{The process and material}

\subsection{Experimental Objectives}

The experimental objectives are to find some usability problems of the CNC machine control panel and select the optimal scheme using multidimensional data synchronous testing analysis method. The validity of this method is verified by a case study. The design schemes are improved so as not only to be easier to use for operator, but also to determine the best scheme for designer.

\subsection{Experiment Equipment}

The 3D modelling software was adopted to complete the design of CNC machine control panel. Four CNC machine control panel interfaces were designed including scheme A, scheme B, scheme C, and scheme D, as shown in Figure 2. The eye movement data was collected using Tobi IT X300 desktop eye tracker. The physiological data was obtained using the portable pulse physiological sensors and skin physiological sensors. The behaviour data came from the behaviour analysis camera. Real-time monitors have been used to ensure the validity of data during the experiment process. The edited stimuli things were to be present in screen. The subjects sat at front of the screen. Their eye movement data could be captured using the eye tracker, which was embedded above the screen. Their finger pulps of index finger and middle finger wore the fingercot of skin conductance sensors. Their earlobes were clamped with ear clamp pulse sensor belonging to the photoelectric pulse sensor. After wearing all the facilities, the subjects were required to keep the static state. To avoid affecting the accuracy of the data, the electrode position couldn't be rubbed and touched. The experimental testing context is shown in Figure 3.

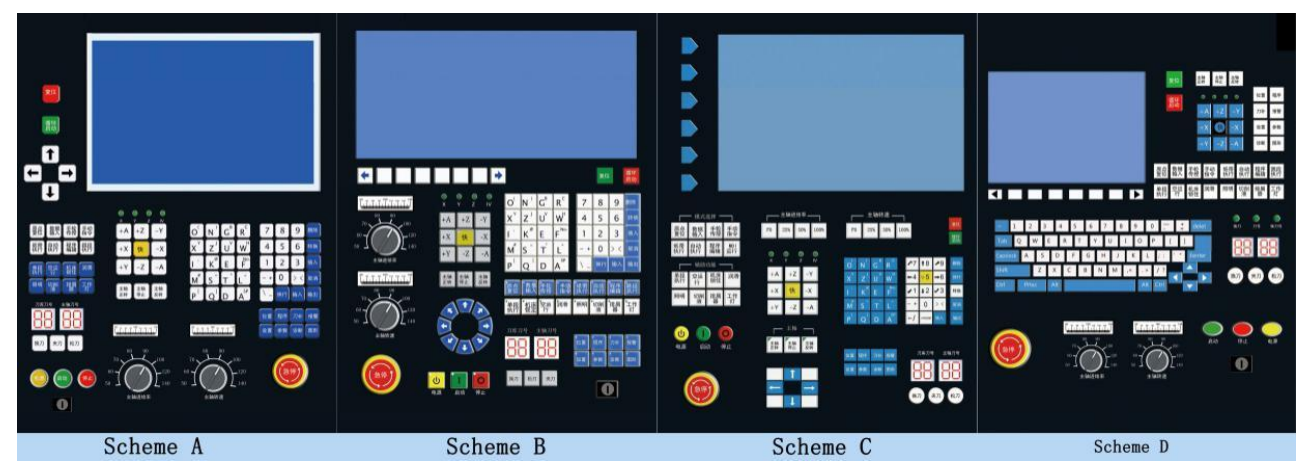

Figure 2. HMI design scheme of CNC machine control panel 


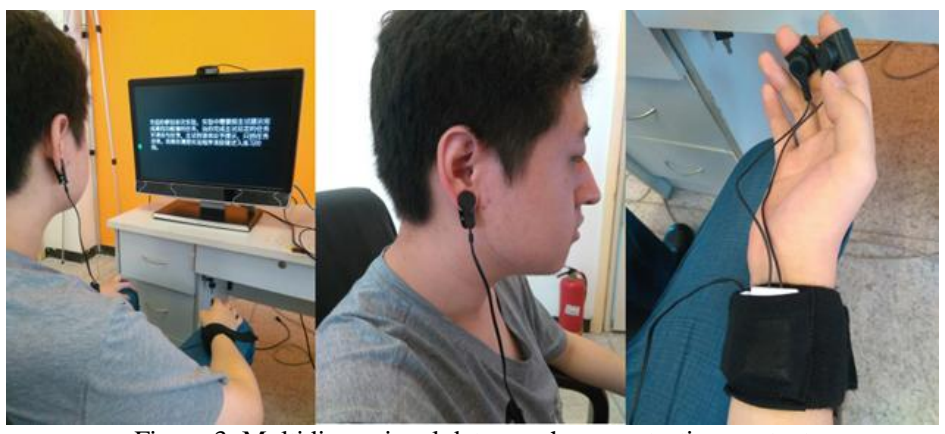

Figure 3. Multidimensional data synchronous testing context

\subsection{Task Design}

Though small sample testing may lead to some deviation, a small sample with five to eight users in usability engineering is enough to collect insights to drive your design [20,21]. There are eight people to be tested in this experiment. They are junior operators engaged in related occupations in a factory. Their corrected visual acuity is normal, and their ages are between 25-40 years old. It is not necessary to have the professional knowledge to operate the CNC machine control panel. The experimental data of the six subjects is valid.

CNC machine control panel was divided into different functional areas. The subjects have finished the tasks designed by experimenters according to the normal operation process. Experimenters told the contents of tasks to subjects with the standard tone and speed. According to the information expressed by experimenters, the subjects found out and operated 7 function keys: Power Supply (PS), Emergency Stop (ES), Zero Point Reset (ZPR), XY, Program (P), Letters (L) and Spindle Feed Rate (SFR) in the four different interfaces of the CNC machine control panel. These 7 keys were selected because they were frequently used in different functional partitions. Examples of operation were as follows: The subjects found the PS key in the interface when the experimenters said, "Please press the function key of the PS". When the subjects found the function key, they oral reported, "I find it". Then, the experimenters told the subjects how to operate this function key. After the task was finished, the experimenters read out the next key name, until the tasks relevant with 7 function keys were completed.

\section{Results and discussions}

The eye movement data, physiological data and behavioral data were collected in real time and were analyzed synchronously. The results and discussions are shown as follows.

\subsection{Eye movement data analysis}

Fixation represents instances of information acquisition and processing, and is closely related to evaluation [12,28,34]. The user's sight stays somewhere and maintains a stable process for a while during the fixation process. In this study, the point where the subject's eyes stay at a fixation object more than $75 \mathrm{~ms}$ (millisecond) is defined as a fixation point. The eye movement data has been recorded including the fixation time, fixation count, average saccade time, saccade count, and visual scanning trajectory. The first fixation time at the function keys of four schemes is shown in Table $1 \sim$ Table 4 .

The total time of the first fixation points in the four schemes is to be compared with each other based on descriptive statistics, as shown in Table 5, unit: second (S). There are some differences with the total time of four schemes at first fixation point. The time spent to view the function keys of the scheme A, scheme B, scheme C, scheme D is different because the interface designs are diverse.

Table 1. The first fixation time at the function keys of scheme A. unit: second(S)

\begin{tabular}{|l|c|c|c|c|c|c|c|}
\hline \multicolumn{1}{|c|}{ Subjects } & PS key & ES key & ZPR key & XY key & P key & L key & SFR key \\
\hline Subjects No.1 & 0.63 & 1.03 & 5.38 & 1.23 & 1.22 & 4.28 & 2.72 \\
\hline Subjects No.2 & 1.47 & 0.77 & 1.95 & 0.83 & 7.28 & 0.2 & 3.12 \\
\hline Subjects No.3 & 1.13 & 1.95 & 3.38 & 1.02 & 5.93 & 0.12 & 1.53 \\
\hline Subjects No.4 & 0.8 & 0.98 & 0.75 & 0.53 & 2.72 & 2.72 & 1.48 \\
\hline Subjects No.5 & 0.42 & 1.08 & 2.25 & 0.22 & 2.53 & 2.53 & 1.92 \\
\hline Subjects No.6 & 1.12 & 0.73 & 1.7 & 0 & 3.05 & 3.05 & 1.58 \\
\hline Average & 0.89 & 1.162 & 2.742 & 0.766 & 3.936 & 1.97 & 2.154 \\
\hline
\end{tabular}


Table 2. The first fixation time at the function keys of scheme B. unit: second(S)

\begin{tabular}{|l|c|c|c|c|c|c|c|}
\hline \multicolumn{1}{|c|}{ Subjects } & PS key & ES key & ZPR key & XY key & P key & L key & SFR key \\
\hline Subjects No.1 & 1.35 & 0.98 & 3.5 & 1.2 & 5.92 & 3.1 & 2.57 \\
\hline Subjects No.2 & 0.75 & 0.78 & 8.02 & 1.47 & 7.8 & 0.78 & 3.38 \\
\hline Subjects No.3 & 0.98 & 0.7 & 5.47 & 1.37 & 4.2 & 0.6 & 2.15 \\
\hline Subjects No.4 & 1.18 & 1 & 1.92 & 0 & 1.25 & 2.52 & 1.58 \\
\hline Subjects No.5 & 2.22 & 1.27 & 2.07 & 1.68 & 1.92 & 0.25 & 3.25 \\
\hline Subjects No.6 & 0.53 & 0.35 & 7.72 & 1.27 & 7.2 & 0.12 & 2.62 \\
\hline Average & 1.296 & 0.946 & 4.196 & 1.144 & 4.218 & 1.45 & 2.586 \\
\hline
\end{tabular}

Table 3. The first fixation time at the function keys of scheme C. unit: second(S)

\begin{tabular}{|l|c|c|c|c|c|c|c|}
\hline \multicolumn{1}{|c|}{ Subjects } & PS key & ES key & ZPR key & XY key & P key & L key & SFR key \\
\hline Subjects No.1 & 0.68 & 2.2 & 1.27 & 1.23 & 4.77 & 0.92 & 9.95 \\
\hline Subjects No.2 & 0.98 & 4.95 & 1.62 & 0.87 & 4.18 & 0.23 & 5.93 \\
\hline Subjects No.3 & 1.12 & 1.03 & 3.87 & 0.82 & 3.87 & 0.48 & 4.2 \\
\hline Subjects No.4 & 1.9 & 0.23 & 3.15 & 0.83 & 5 & 3.15 & 9.55 \\
\hline Subjects No.5 & 0.5 & 0.58 & 3.98 & 0.12 & 20.62 & 0.92 & 3.63 \\
\hline Subjects No.6 & 1.2 & 0.67 & 1.23 & 0.52 & 5.18 & 0.05 & 4.03 \\
\hline Average & 1.036 & 1.798 & 2.778 & 0.774 & 7.688 & 1.14 & 6.652 \\
\hline
\end{tabular}

Table 4. The first fixation time at the function keys of scheme D. unit: second(S)

\begin{tabular}{|l|c|c|c|c|c|c|c|}
\hline \multicolumn{1}{|c|}{ Subjects } & PS key & ES key & ZPR key & XY key & P key & L key & SFR key \\
\hline Subjects No.1 & 7.25 & 3.1 & 4.47 & 0.2 & 13.37 & 1.57 & 2.28 \\
\hline Subjects No.2 & 2.1 & 3.22 & 4.92 & 0.8 & 4.07 & 0.82 & 1.92 \\
\hline Subjects No.3 & 4.53 & 1.02 & 3.48 & 0.73 & 3.68 & 1.6 & 3.07 \\
\hline Subjects No.4 & 1.39 & 1.27 & 2.3 & 0.92 & 4.53 & 0.37 & 4.53 \\
\hline Subjects No.5 & 4.12 & 0.9 & 1.62 & 0 & 1.98 & 0.78 & 2.17 \\
\hline Subjects No.6 & 2.1 & 1.03 & 3.25 & 1.27 & 2.77 & 0.72 & 2.28 \\
\hline Average & 3.878 & 1.902 & 3.358 & 0.53 & 5.526 & 1.028 & 2.794 \\
\hline
\end{tabular}

Table 5. Total fixation time of four schemes (descriptive statistics) unit: second(S)

\begin{tabular}{|c|c|c|c|}
\hline Interface scheme & Mean & Std. Deviation & N \\
\hline Total Fixation Time of Scheme A & 13.2217 & 2.80888 & 6 \\
\hline Total Fixation Time of Scheme B & 16.4983 & 4.95883 & 6 \\
\hline Total Fixation Time of Scheme C & 20.3683 & 6.25059 & 6 \\
\hline Total Fixation Time of Scheme D & 18.0833 & 7.38008 & 6 \\
\hline
\end{tabular}

The first fixation time trend of each function key is plotted as shown in Figure 4. A, B, C, and D represent the first fixation time point curve of four different interface schemes which comprise scheme A, scheme B, scheme C and scheme D. The results display that the trends of the five keys interfaces including ES key, ZPR key, XY key, P key, and Letter key are consistent; however, the trends of PS key and SFR key are very different. Therefore, we would discuss the eye movement trajectories analysis of the PS key and SFR key.

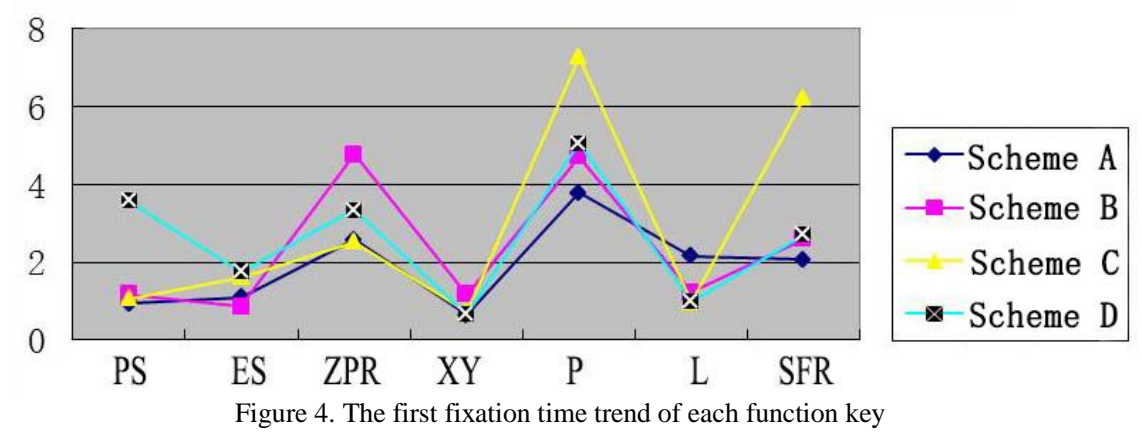

The three subject's eye movement trajectories of PS keys in scheme A and scheme D are shown in Figure 5. It is seen that the search path of PS key in the interface scheme A is shorter than that of the interface scheme D. The information 
search pattern of interface scheme A is from top to bottom. It can be inferred that the design of interface scheme A is more reasonable, since the subjects can search its PS key position within a short period of time. The power key of scheme A is set in the lower left corner; the position effect of interface scheme A is obvious. On the contrary, the PS key of interface scheme $\mathrm{D}$ is located on the last search area and is hard to be fixated. As an initial function key of the interface, the location factor should be considered.

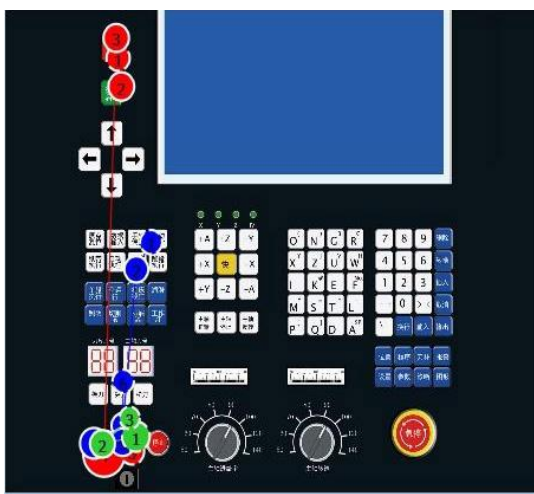

Scheme A

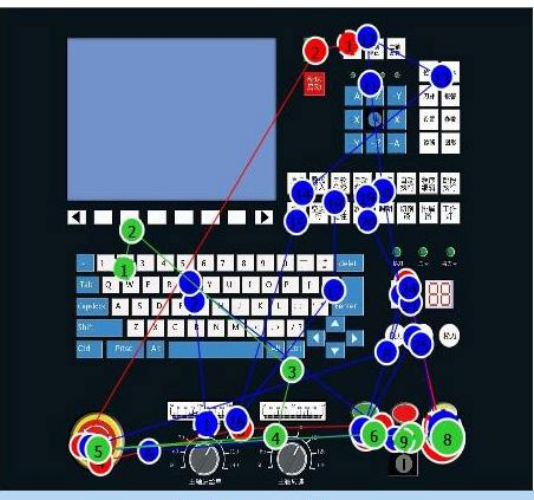

Scheme D

Figure 5. Eye movement trajectories of three test persons for the PS key

Furthermore, we also make a comparative analysis for the eye movement trajectories of SFR keys. There is a significant difference in search time between the interface scheme $\mathrm{A}$ and interface scheme $\mathrm{C}$. The eye movement trajectories of three subjects in two schemes are shown in Figure 6.

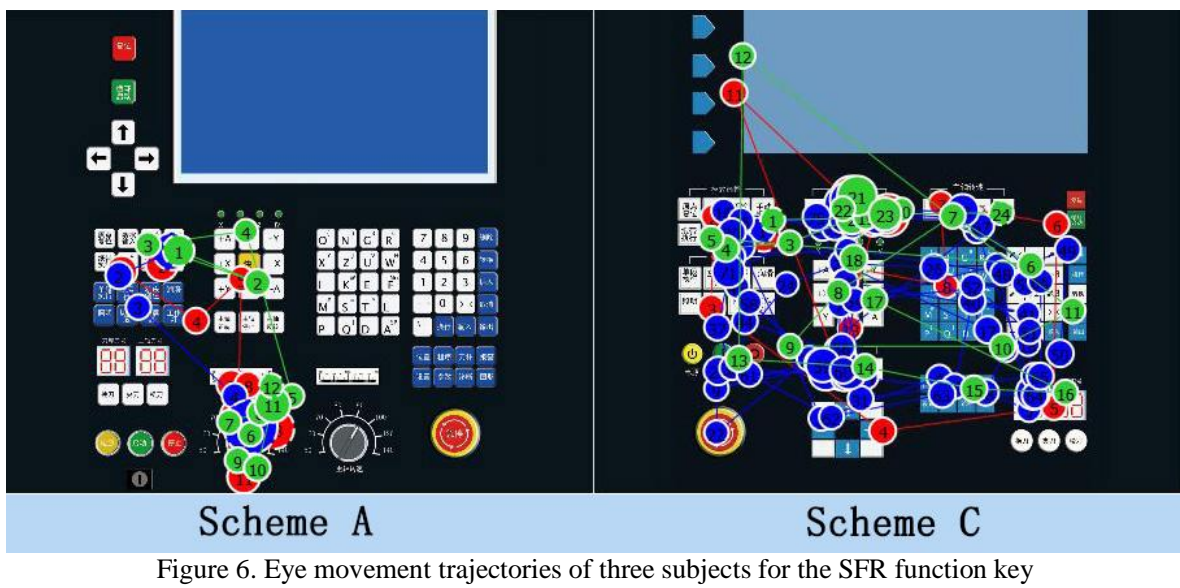

The search path of SFR key in the scheme A is shorter than that of the scheme C, and there are so many saccade counts in scheme C. The eye movement trajectories show that SFR key in scheme A is easier to use than scheme C. The spindle feed rate in the interface of scheme $\mathrm{A}$ is designed as a very normal dashboard model. But the spindle feed rate in the interface of scheme $\mathrm{C}$ is substituted for the detailed digital module. This shape of SPR is similar to other function keys, as shown in Figure 7. When the subjects search the function keys, the circular dashboard model is easier to find. So, there is the obvious effect of key's shape on its operating efficiency.

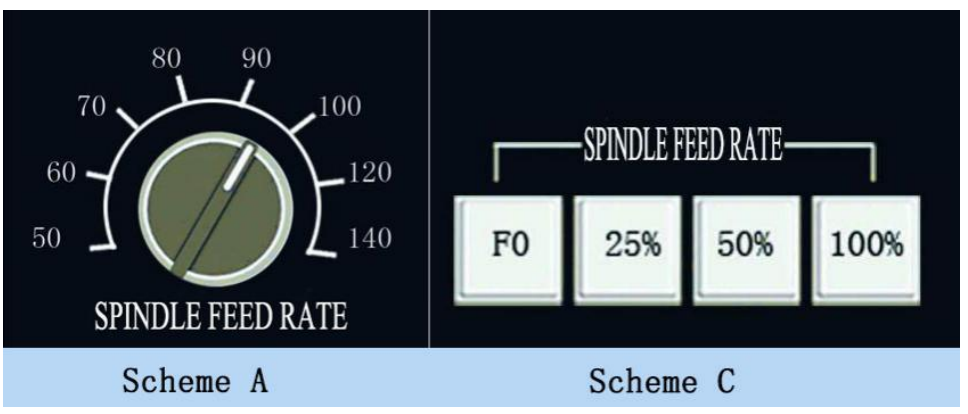

Figure 7. Form design for spindle feed rate key of Scheme A and Scheme C 


\subsection{Physiological data analysis}

Heart rate variability (HRV) data is obtained using physiological sensors. High Frequency (HF) of HRV which represents parasympathetic activity is considered and it's an index of cardiac parasympathetic control [5]. Low Frequency (LF) of HRV is mainly affected by sympathetic modulation [19]. LF/ HF can be used as quantitative index for evaluating the balance of cardiac vagal and sympathetic nerve.

Since the values of LF and HF are directly affected by the total power spectrum (TP), especially in the short-term analysis. The TP, LF and HF values in different states are different. The total power spectrum shows the variability of the total signal of autonomic nervous activity. If compared with the absolute values directly, researchers often obtain some wrong conclusions. The comparison should be done after these values are normalized. The HRV spectrum analysis has two relevant indexes: normalized low frequency (LFnorm) and normalized high frequency (HFnorm). Hfnorm and Lfnorm reflect the changes of parasympathetic nerve and sympathetic nerve more directly and their formula (NASPE, 1996) [32] is shown as:

$$
L F(\text { or } H F)_{n o r m}=\frac{100 \times L F(\text { or } H F)}{(T P-V L F)} \quad \text { (n.u.) }
$$

The results of HFnorm index to be analyzed with variances show that there are some differences among the four interfaces (as shown in Figure 8). Both the interface $\mathrm{C}$ and interface D have caused higher parasympathetic nerve activity. Compared with the LFnorm of the low frequency band power (as shown in Figure 9), the results of LFnorm index to be analyzed with variances indicate that there are also some differences among the four interfaces. The sympathetic nerve activity gradually decreases by interfaces scheme A, scheme B, scheme C, and scheme D. Pfister et al. [25] showed that the pleasure level induced by good operational feedback increased the HR of users. Rainville et.al. [27] showed that the increase in heart rate observed in fear, happiness, sadness and decrease in high frequency (HF). Kop, Synowski, and Newell [15] indicated that positive emotions like happiness recall resulted in the increased LF. Emotion experience, such as happiness or unhappiness, is the main emotional experience when the users operate the human computer/machine interface. Both indexes of HFnorm and LFnorm indicate that the experience of interface $\mathrm{C}$ and interface $\mathrm{D}$ is not better than that of interface A and interface B.

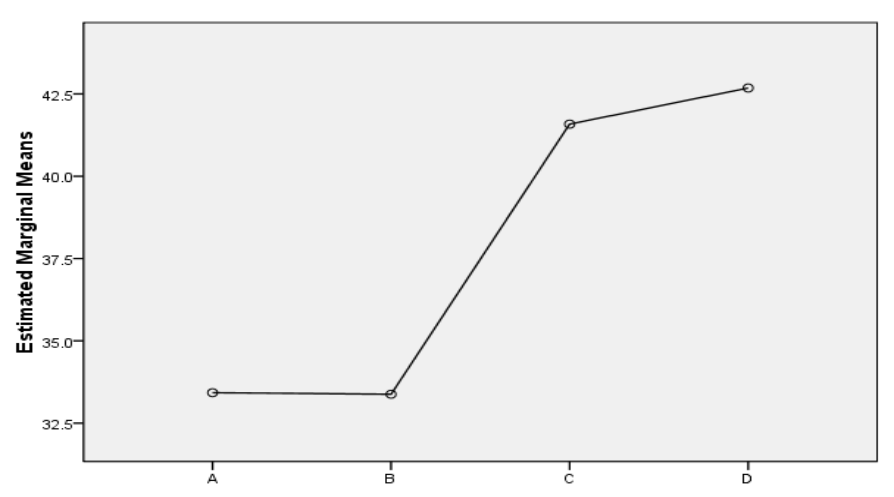

Figure 8. HFnorm means of four design schemes

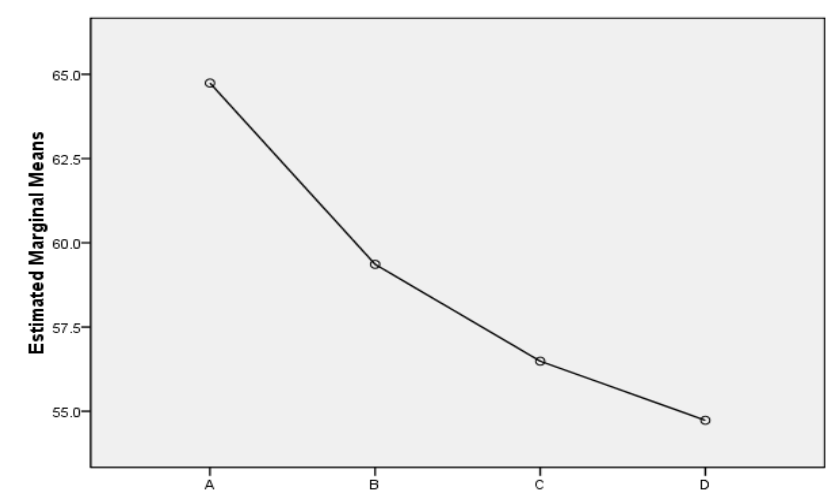

Figure 9. LFnorm means of four design schemes 


\subsection{Behavioral data}

The statistics of behavior data show the total time that the subjects have spent to finish all tasks of the four interfaces. The trends of total time to finish all tasks are shown in Figure 10. It is found that the total time to finish the tasks of interface scheme $\mathrm{C}$ is the longest, and the total time to finish the tasks of interface scheme $\mathrm{A}$ is the shortest, which agrees with the results of eye movement analysis and physiological analysis.

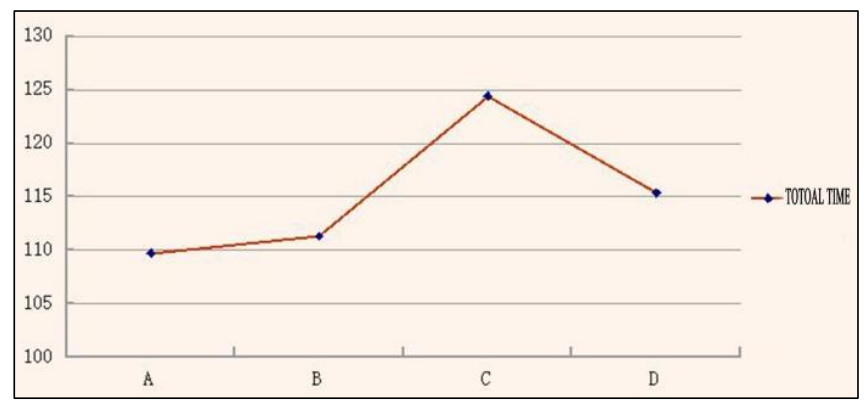

Figure 10. Total task completion time trend chart (unit: S)

As above mentioned, the implications can be analyzed from physiological data, eye movement data and behavior analysis data. We can infer that the interface scheme A has displayed the highest availability and it brings a better experience to users. While both interface C and interface D are in the worst state of availability, the influence factors are identified and they are needed to be modified urgently.

\section{Conclusions}

The multidimensional data synchronous testing analysis method is used to evaluate the HMI design schemes of CNC machine control panel. Due to the real-time and synchronization of data acquisition, the mutual validation of multidimensional data makes the results more objectively. The study shows that the interface scheme A has a better user experience and its availability is best. The operation efficiency of interface scheme $\mathrm{C}$ and $\mathrm{D}$ is low and their user experience is not good. The result indicates that the interface layout and button shape of CNC machine control panel have significant influence on the user's operation efficiency as well as experience. Then, the designer can improve the design schemes and iterative optimize the HMI design of CNC machine control panel. The method of multidimensional data synchronous testing analysis has widely application prospects in the process of design and development human-machine interface.

There are some limitations to the evaluation of HMI of CNC machine control panel. The subjects were limited to 25-40 years old and the data was collected from a small number of participants. In the next phase of the study, we will make improvements to these deficiencies.

\section{Acknowledgements}

We would like to thank all colleagues and teachers who help for our work: Kingfar International Inc and Qingju Wang, Shengxing Ban supported our experimental data acquisition. Ministry of Education Humanities and Social Sciences Research Youth Fund Project [No. 15YJCZH034] that partially supported our research work.

\section{References}

1. A. Açık, D. E. Barkana, G. Akgün, A. E. Yantaç, and Ç. Aydın, "Evaluation of a surgical interface for robotic cryoablation task using an eye-tracking system," International Journal of Human-Computer Studies, vol. 95, no.C, pp.39-53, 2016

2. J. Akyeampong, S. Udoka, G. Caruso, and M. Bordegoni, "Evaluation of hydraulic excavator human-machine interface concepts using nasa tlx," International Journal of Industrial Ergonomics, vol. 44, no.3, pp.374-382, 2014

3. J. L. Andreassi, "Psychophysiology: Human behavior and physiological response (3rd ed.)," Lawrence Erlbaum Associates, Mahwah, New Jersey, United States, 1995

4. G. Andreoni, M. Mazzola, D. Zambarbieri, L. Forzoni, S. D'Onofrio, S. Viotti, G. C. Santambrogio, and G. Baselli,"Motion analysis and eye tracking technologies applied to portable ultrasound systems user interfaces evaluation," in International Conference on Computer Medical Applications (ICCMA), vol.58, pp.1-6, Sousse,Tunisia,January 2013

5. G. G. Berntson, J. T. J. Bigger, D. L. Eckberg, P. Grossman, P. G. Kaufmann, M. Malik, H. Nagaraja, S. Porges, P. Saul, P. Stone, and M. van der Molen, "Heart rate variability: Origins, methods, and interpretive caveats," Psychophysiology, vol.34, no.6, pp.623-648,1997

6. A. Darwish and E. Bataineh, "Eye tracking analysis of browser security indicators," in International Conference on Computer 
Systems and Industrial Informatics, pp.1-6, Sharjah, United Arab Emirates, 2012

7. A. C. Dirican, and M.Göktürk, "Psychophysiological measures of human cognitive states applied in human computer interaction," Procedia Computer Science, vol. 3, no.1, pp.1361-1367, 2011

8. C. Guo, "Methods towards Synchronous Recording and Analysis of Eye Movements and Electroencephalograph," Tsinghua University, Beijing. 2012.

9. J. Gao, Q. C. Zhao, F. Zhou, Q. J. Wang, F. Liu, and Z. Q. Li, "Study on driver behavior using "Kingfar synchronization" approach-To explore driver's visual behavior when preparing for driving." Journal of Beijing Institute of Technology, vol. 20, pp.260-265, 2011

10. L. Giraudet, J. P. Imbert, M. Bérenger, S. Tremblay, and M. Causse, "The neuroergonomic evaluation of human machine interface design in air traffic control using behavioral and EGG/ERP measures," Behavioural Brain Research, vol. 294, no.5, pp.246-253, 2015

11. F. Guo, Y. Cao, Y. Ding and Q. U. Qingxing, "The Emotional Experience of E-commerce Websites Using Multi-mode Measurements," China Journal of Information Systems, vol.1, pp.24-36, 2013.

12. L. A. Granka, T. Joachims and G. Gay, "Eye-tracking analysis of user behavior in WWW search," in International ACM SIGIR Conference on Research and Development in Information Retrieval, pp.478-479, 2004

13. J. S. Ha, and P. H. Seong, "A human-machine interface evaluation method: a difficulty evaluation method in information searching (demis)," Reliability Engineering \& System Safety, vol. 94, no.10, pp. 1557-1567, 2009

14. C. H. Huang, "human-machine interface," Computer Science and Communications Dictionary. Springer US, New York, USA, 2012

15. W. J. Kop, S. J. Synowski and M. E. Newell, "Autonomic nervous system reactivity to positive and negative mood induction: the role of acute psychological responses and frontal electrocortical activity," Biological Psychology, vol.86, no.3, pp.230-238, 2011

16. Y. Lin and W. J. Zhang, "Combining eye movement and hand movement measures for evaluating human-machine interfaces," in IEEE International Conference on System, Man and Cybernetics, vol.2, pp.1089-1096, Washington, DC, USA, October 2003

17. H. Masaki, M. Ohira, H. Uwano, and K. Matsumoto, "A Quantitative Evaluation on the Software Use Experience with Electroencephalogram. Design, User Experience, and Usability. Theory, Methods, Tools and Practice," Springer-Verlag, Berlin Heidelberg, Germany, 2011

18. D. C. Montgomery, "Design and Analysis of Experiments," Wiley, New York, USA, 2000.

19. N. Montano, R. Gnecchi, A. Porta, F. Lombardi, M. Pagani and A. Mallani, "A power spectrum analysis of heart rate variability to assess the changes in sympathovagal balance during graded orthostatic tilt," Circulation, vol.90, pp.1826-1831, 1984

20. J. Nielsen, "How many test users in a usability study?" from https://www.nngroup.com/articles/how-many-test-users, June 4, 2012

21. J. Nielsen, "Why you only need to test with 5 users?" from https:// https://www.nngroup.com/articles/why-you-only-need-totest-with-5-users, March 19, 2000

22. Y. Niu, C. Xue, N. Peng, L. Zhou, W. Wu, R. Guan and T. Jin, "Cognition and evaluation of digital interface layouts based on event-related potential technique,” Journal of Southeast University (Natural Science Edition), vol.46, pp.470-475, 2016

23. B. Park, "Psychophysiology as a Tool for HCI Research: Promises and Pitfalls," Human-Computer Interaction. New Trends, Springer-Verlag, Berlin Heidelberg, Germany, 2009

24. H. Park, and H. C. Moon, "Design evaluation of information appliances using augmented reality-based tangible interaction," Computers in Industry, vol.64, no.7, pp.854-868, 2013

25. H. R. Pfister, S. Wollstädter and C. Peter, "Affective responses to system messages in human-computer-interaction: Effects of modality and message type," Interacting with Computers, vol. 23, no.4, pp. 372-383, 2011

26. P. Ramakrisnan, A. Jaafar, F. H. A. Razak, and D. A. Ramba, "Evaluation of user interface design for leaning management system (lms): investigating student's eye tracking pattern and experiences 5 ," Procedia - Social and Behavioral Sciences, vol.67, pp.527-537, 2012.

27. P. Rainville, A. Bechara, N. Naqvi and A. R. Damasio, "Basic emotions are associated with distinct patterns of cardiorespiratory activity," International Journal of Psychophysiology, vol.61, no.1, pp.5-18, 2006

28. K. Rayner, "Eye movements in reading and information processing: 20 years of research," Psychological Bulletin, vol.124, no.3, pp.372-422, 1998

29. J. F. Reintjes, "Numerical control: making a new technology," Oxford University Press, New York, USA, 1991.

30. A. Rogalska, and P. Napieralski, "A problem of assessing visual comfort in virtual reality applications," In book: Computer Game Innovations, pp.43-53, 2016

31. A. Sinharay, D. Chatterjee, and A. Sinha, "Evaluation of Different Onscreen Keyboard Layouts Using EEG Signals," in IEEE International Conference on Systems, Man, and Cybernetics, vol. 8215, pp.480-486, Hong Kong, China, October 2014

32. Task Force of the European Society of Cardiology (ESC) and the North American Society of Pacing Electrophysiology (NASPE), "Heart rate variability: Standards of measurement, physiological interpretation, and clinical use," European Heart Journal, vol. 17, no.3, pp.354-381, 1996.

33. K. Uggeldahl, C. Jacobsen, T. H. Lundhede, and S. B. Olsen, "Choice certainty in discrete choice experiments: will eye tracking provide useful measures?" Journal of Choice Modelling, vol.20, pp.35-48, 2016

34. N. H. M. Zain, F. H. A. Razak, A. Jaafar, and M. F. Zulkipli, "Eye Tracking in Educational Games Environment: Evaluating User Interface Design through Eye Tracking Patterns," International Conference on Visual Informatics: Sustaining Research and Innovations, pp.64-73, Springer-Verlag, Berlin Heidelberg, 2011

35. S. R. Zhou, S. Jeon, H. Sim, and W. Lee, "User interface evaluation method using eye tracking," in the 18th IEEE International Symposium on Consumer Electronics, pp. 1-2, JeJu, Korea, June 2014 
Jinhua Dou is currently a Ph.D. candidate in Computer Science and Technology at the University of Science and Technology Beijing, Beijing, China. She has also worked with school of Art \& Design, Tianjin University of Technology, where she is an associate professor. Dou received a Master's degree in design art from Hunan University, Changsha, China. Her research interests include Human-Computer Interaction, Human Factors Engineering, Information Visualization and Product System Design.

Lei Zhang is the associate professor of Tianjin University of Technology. Zhang received his M.S. degrees in Design Art from Beijing Institute of Technology. His research interests include Ergonomics and Industrial Design.

Qichao Zhao is vice-chairman of Virtual Reality Specialized Committee in China User Experience Alliance. He has been served as CEO of Kingfar International Inc and his research interests include Human Factors Engineering, User Experience and Product Service System Development.

Qin Pei received her bachelor's degree from Tianjin University of Technology. Her research interests include Ergonomics and Industrial Design.

Jingyan Qin is a full professor at University of Science and Technology Beijing. She is the Ph.D. supervisor in Big Data Information Visualization and Interaction Design at Computer Science School of USTB, and she is the director of HCI and design for Sustainability Research Center at Industrial Design Department, USTB. She is selected as the Fellow of New Century Talents Plan of Ministry of Education of China in 2013. And she is the committee member of Ministry of Culture, P.R. China, the Senior Fellow of China Computer Federation, the committee member of China Computer Federation and YOCSEF, ACM, executive director of Beijing Green Design Association, China Information and Interaction Design Association, Fellow of Beijing Design Federation, the academic committee member of Information and Interaction Design Committee. Her research and education focus on Interaction Design, Web Design, Sustainable Innovation Design, Digital Entertainment Design and New Media Art. 